\title{
Warm Spitzer Photometry of the Transiting Exoplanets Corot-1 and Corot-2 at Secondary Eclipse
}

\section{Citation}

Deming, Drake, Heather Knutson, Eric Agol, Jean-Michel Desert, Adam Burrows, Jonathan J. Fortney, David Charbonneau, et al. 2010. "WARMSPITZERPHOTOMETRY OF THE TRANSITING EXOPLANETS CoRoT-1 AND CoRoT-2 AT SECONDARY ECLIPSE." The Astrophysical Journal 726 (2): 95. https://doi.org/10.1088/0004-637x/726/2/95.

\section{Permanent link}

http://nrs.harvard.edu/urn-3:HUL.InstRepos:41397438

\section{Terms of Use}

This article was downloaded from Harvard University's DASH repository, and is made available under the terms and conditions applicable to Other Posted Material, as set forth at http:// nrs.harvard.edu/urn-3:HUL.InstRepos:dash.current.terms-of-use\#LAA

\section{Share Your Story}

The Harvard community has made this article openly available.

Please share how this access benefits you. Submit a story.

\section{Accessibility}




\title{
WARM SPITZER PHOTOMETRY OF THE TRANSITING EXOPLANETS CoRoT-1 AND CoRoT-2 AT SECONDARY ECLIPSE
}

\author{
Drake Deming ${ }^{1}$, Heather Knutson $^{2,10}$, Eric Agol ${ }^{3}$, Jean-Michel Desert $^{4}$, Adam Burrows 5 , Jonathan J. Fortney ${ }^{6}$, \\ David Charbonneau ${ }^{4}$, Nicolas B. Cowan ${ }^{3,7,11}$, Gregory Laughlin ${ }^{6}$, Jonathan Langton $^{8}$, Adam P. Showman ${ }^{9}$, \\ AND NiKOLE K. LEWIS ${ }^{9}$ \\ ${ }^{1}$ Planetary Systems Laboratory, NASA's Goddard Space Flight Center, Greenbelt, MD 20771, USA \\ ${ }^{2}$ Department of Astronomy, University of California at Berkeley, Berkeley, CA 94720, USA \\ ${ }^{3}$ Department of Astronomy, University of Washington, Box 351580, Seattle, WA 98195, USA \\ ${ }^{4}$ Harvard-Smithsonian Center for Astrophysics, Cambridge, MA 02138, USA \\ ${ }^{5}$ Department of Astrophysical Sciences, Princeton University, Princeton, NJ 05844, USA \\ ${ }^{6}$ Department of Astronomy and Astrophysics, University of California at Santa Cruz, Santa Cruz, CA 95064, USA \\ ${ }^{7}$ Department of Physics \& Astronomy, Northwestern University, 2131 Tech Drive, Evanston, IL 60208, USA \\ ${ }^{8}$ Department of Physics, Principia College, Elsah, IL 62028, USA \\ ${ }^{9}$ Lunar and Planetary Laboratory, University of Arizona, Tucson, AZ 85721, USA \\ Received 2010 May 4; accepted 2010 November 2; published 2010 December 20
}

\begin{abstract}
We measure secondary eclipses of the hot giant exoplanets CoRoT- 1 at 3.6 and $4.5 \mu \mathrm{m}$, and CoRoT-2 at $3.6 \mu \mathrm{m}$, both using Warm Spitzer. We find that the Warm Spitzer mission is working very well for exoplanet science. For consistency of our analysis we also re-analyze archival cryogenic Spitzer data for secondary eclipses of CoRoT-2 at 4.5 and $8 \mu \mathrm{m}$. We compare the total data for both planets, including optical eclipse measurements by the CoRoT mission, and ground-based eclipse measurements at $2 \mu \mathrm{m}$, to existing models. Both planets exhibit stronger eclipses at 4.5 than at $3.6 \mu \mathrm{m}$, which is often indicative of an atmospheric temperature inversion. The spectrum of CoRoT- 1 is best reproduced by a $2460 \mathrm{~K}$ blackbody, due either to a high altitude layer that strongly absorbs stellar irradiance, or an isothermal region in the planetary atmosphere. The spectrum of CoRoT- 2 is unusual because the $8 \mu \mathrm{m}$ contrast is anomalously low. Non-inverted atmospheres could potentially produce the CoRoT-2 spectrum if the planet exhibits line emission from $\mathrm{CO}$ at $4.5 \mu \mathrm{m}$, caused by tidal-induced mass loss. However, the viability of that hypothesis is questionable because the emitting region cannot be more than about 30\% larger than the planet's transit radius, based on the ingress and egress times at eclipse. An alternative possibility to account for the spectrum of CoRoT-2 is an additional opacity source that acts strongly at wavelengths less than $5 \mu \mathrm{m}$, heating the upper atmosphere while allowing the deeper atmosphere seen at $8 \mu \mathrm{m}$ to remain cooler. We obtain a similar result as Gillon et al. for the phase of the secondary eclipse of CoRoT-2, implying an eccentric orbit with $e \cos (\omega)=-0.0030 \pm 0.0004$.
\end{abstract}

Key words: eclipses - planetary systems - techniques: photometric

Online-only material: color figures

\section{INTRODUCTION}

An especially interesting class of giant extrasolar planets, the "very hot Jupiters" (VHJs), orbit extremely close to solartype stars, within $0.03 \mathrm{AU}$ in several cases. The temperature structure in the atmosphere of such a planet is likely to be significantly perturbed by the strong stellar irradiation. Absorption of stellar radiation is one possible energy source that may drive atmospheric temperature inversions. Temperature inversions with height appear to be common in hot Jupiter atmospheres; they occur over a wide range of stellar irradiation level (Knutson et al. 2008; Machalek et al. 2008; Christiansen et al. 2010; Todorov et al. 2010), but are not well understood. The emergent spectra of VHJs are an important key to this problem. The emergent spectrum of a transiting planet can often be measured by observing the decrease in total light as the planet passes behind the star during secondary eclipse (Charbonneau et al. 2005; Deming et al. 2005). Eclipses of the VHJs offer the opportunity to determine their emergent spectra at wavelengths as short as visible light (Alonso et al. 2009a; Snellen et al. 2009). Fortunately, VHJs have high transit probabilities, and

\footnotetext{
${ }^{10}$ Miller Research Fellow.

${ }^{11}$ Currently CIERA Fellow.
}

are represented by transiting planets such as WASP-12 (Hebb et al. 2009), WASP-19 (Hebb et al. 2010), CoRoT-1 (Barge et al. 2008), and CoRoT-2 (Alonso et al. 2008).

The CoRoT planets are particularly important in the study of VHJ temperature structure. Their emergent flux has been measured at secondary eclipse using infrared (IR) wavelengths, and also in the visible by the CoRoT mission. The currently available secondary eclipse measurements for the CoRoT planets are summarized in Table 1, including the results from this paper. While eclipses of CoRoT-2 have been measured at $4.5 \mu \mathrm{m}$ and $8.0 \mu \mathrm{m}$ using the Spitzer Space Telescope (Gillon et al. 2010), no Spitzer measurements have been reported for CoRoT-1. In this paper, we report measurements of CoRoT-1 using Warm Spitzer (Deming et al. 2007) at 3.6 and $4.5 \mu \mathrm{m}$, and we complete Spitzer's measurement of CoRoT-2 by adding the $3.6 \mu \mathrm{m}$ observation. These additional data allow us to address the existence and nature of the inversion phenomenon in these planets. Moreover, because we measure near the peak of the VHJ's spectral energy distribution, we can speak to whether the visible wavelength eclipse measurements are sensing primarily thermal radiation, as opposed to reflected light.

Our results, together with those of Hebrard et al. (2010), are among the first to be reported for transiting exoplanets using Warm Spitzer. The Warm phase of Spitzer refers to operation of 
Table 1

Summary of Secondary Eclipse Measurements for CoRoT-1 and CoRoT-2

\begin{tabular}{clcc}
\hline \hline Planet & Wavelength & Eclipse Depth & Reference \\
\hline CoRoT-1 & $0.60(0.42) \mu \mathrm{m}$ & $0.016 \% \pm 0.006 \%$ & Alonso et al. (2009b) \\
$\ldots$ & $0.71(0.25)$ & $0.0126 \% \pm 0.0033 \%$ & Snellen et al. (2009) \\
$\ldots$ & $2.10(0.02)$ & $0.278 \%+0.043 \%$ & Gillon et al. (2009a) \\
$\ldots$ & $2.15(0.32)$ & $0.336 \% \pm 0.042 \%$ & Rogers et al. (2009) \\
$\ldots$ & $3.6(0.75)$ & $0.415 \% \pm 0.042 \%$ & This paper \\
$\ldots$ & $4.5(1.0)$ & $0.482 \% \pm 0.042 \%$ & This paper \\
CoRoT-2 & $0.60(0.42) \mu \mathrm{m}$ & $0.006 \% \pm 0.002 \%$ & Alonso et al. (2009a) \\
$\ldots$ & $0.71(0.25)$ & $0.0102 \% \pm 0.002 \%$ & Snellen et al. (2010) \\
$\ldots$ & $2.15(0.32)$ & $0.16 \% \pm 0.09 \%$ & Alonso et al. (2010) \\
$\ldots$ & $3.6(0.75)$ & $0.355 \% \pm 0.020 \%$ & This paper \\
$\ldots$ & $4.5(1.0)$ & $0.510 \% \pm 0.042 \%$ & Gillon et al. (2010) \\
$\ldots$ & $4.5(1.0)$ & $0.500 \% \pm 0.020 \%$ & This paper \\
$\ldots$ & $8.0(2.9)$ & $0.41 \% \pm 0.11 \%$ & Gillon et al. (2010) \\
$\ldots$ & $8.0(2.9)$ & $0.446 \% \pm 0.10 \%$ & This paper - log ramp \\
\hline
\end{tabular}

the observatory after the loss of cryogen, with only the 3.6 and $4.5 \mu \mathrm{m}$ channels of the Infrared Array Camera (IRAC; Fazio et al. 2004) instrument remaining operational. The InSb detectors used at these wavelengths are now functioning at a temperature of approximately 29 Kelvins, cooled by passive radiation. This very different operating temperature regime may have significant implications for the observatory performance as regards high precision photometry. Therefore, we comment on the performance of the observatory, within the limits allowed by the fact that we have observed relatively faint stars.

In Section 2, we describe the observations, aperture photometry, and linear regression procedures to derive eclipse depths and central phases. Section 3 discusses the implications of our results for the orbital and atmospheric properties of these giant CoRoT planets, and in the Appendix we discuss some details concerning the performance of the Warm Spitzer observatory for this type of exoplanet science.

\section{OBSERVATIONS AND PHOTOMETRY}

\subsection{CoRoT-1}

We observed CoRoT-1 at $4.5 \mu \mathrm{m}$ on 2009 November 23, starting at 11:06 UT (orbital phase 0.380), for a duration of 465.7 minutes, yielding $88830 \mathrm{~s}$ exposures. Among transiting systems, CoRoT-1 is relatively faint, having $V=13.6$ and $K=12.1$, and a short orbital period of $P=1.509$ days (Barge et al. 2008). We observed this system at $3.6 \mu \mathrm{m}$ on 2009 November 26, starting at 11:30 UT (orbital phase 0.379) for the same duration, and the same exposure time per frame. The CoRoT-1 observations at both wavelengths used full frame $(256 \times 256$ pixel $)$ mode. Following the eclipse observations, we acquired 9 minutes of additional data (17 exposures) by offsetting the telescope to view blank sky using the same detector pixels as for CoRoT-1.

The detectors in the Warm mission are significantly affected by an artifact called column pull-down, ${ }^{12}$ wherein the presence of a bright star reduces the signal level for an entire detector column. This, as well as other artifacts, is significantly mitigated in the cBCD files produced by Spitzer's pipeline processing. However, neither CoRoT-1 nor CoRoT-2 lie on columns affected by pull-down, and in any case we would want to remove any such artifacts as an integral part of our photometry, so

\footnotetext{
12 See http://ssc.spitzer.caltech.edu/irac/warmfeatures/
}

that we could fully judge their impact. We therefore extracted photometry using the basic calibrated data (BCD) files produced by version S18.12.0 of the Spitzer pipeline, not the cBCD files. We calculated orbital phase using the UTC-based HJD values for the start of each observation from the FITS headers of the $\mathrm{BCD}$ files, and we correct the values to the time of mid-exposure.

As a first step, we stack the blank sky images and medianfilter each pixel in time to construct an average blank sky frame. We subtract this sky frame from each CoRoT-1 image immediately after reading each BCD file. In principle, this subtraction of a sky-nod will remove the background radiation, but we nevertheless fit and remove residual background anyway, as described below. Although the true sky background should be constant to an excellent approximation, we find that the background does vary significantly from frame to frame. This is one significant difference from the cryogenic mission, as we discuss in the Appendix.

We locate and correct energetic particle events by comparing the time history of each pixel to a 5-point median filter of that pixel intensity versus time, and we replace $>4 \sigma$ outliers with the median value. The fraction of pixels we correct varies between $0.45 \%$ and $0.55 \%$, depending on which planet and wavelength are analyzed. We perform aperture photometry on the images, after first applying corrections for variations in pixel solid angle, and for slightly different flat-field response for point sources versus extended sources. ${ }^{13}$ Prior to subtracting the residual background and performing aperture photometry, we convert the pixel intensities to electrons, using the calibration information given in the FITS headers. This facilitates the evaluation of the photometric errors.

Our photometry code locates the centroid of the stellar pointspread function (PSF) by fitting a symmetric two-dimensional Gaussian to the PSF core (Agol et al. 2010). We calculate the flux within a centered circular aperture, of variable radius, using radii of 2.0 to 4.5 pixels, in 0.5 pixel steps. To determine the residual background intensity, we fit a Gaussian to a histogram of pixel intensities for each frame. The center histogram bin, defined to fractional precision by the Gaussian fit, is adopted as the residual background intensity. Subtracting the resultant background from the raw aperture photometry, yields 6 photometric time series for the star corresponding to aperture radii from 2.0 to 4.5 pixels. We tabulate the magnitude of the point-to-point scatter in our

13 See Sections 5.3 and 5.6.2 of the IRAC Data Handbook, V3.0. 


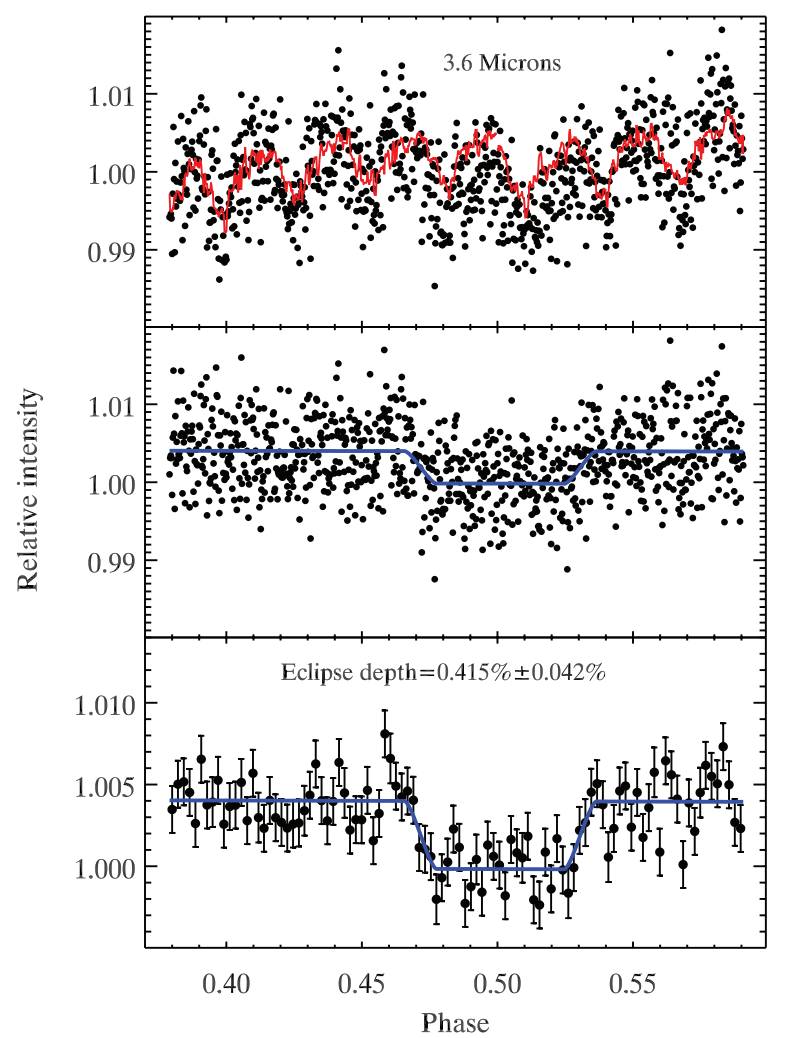

Figure 1. Upper panel: photometry of CoRoT-1 vs. orbital phase, at $3.6 \mu \mathrm{m}$ (points), with the decorrelation function overplotted (red line). Middle panel: photometry after correction with the decorrelation function, with the best-fit eclipse curve overlaid (blue line). Bottom panel: decorrelated photometry binned to a resolution of approximately 0.002 in orbital phase (100 bins), with the bestfit eclipse curve overlaid (blue line). The error bars are based on the scatter of individual points within each bin. The best-fit central phase is $0.5012 \pm 0.0024$. (A color version of this figure is available in the online journal.)

photometry, and errors in our final results, as a function of aperture radius. We find that both the scatter and final parameter errors depend only weakly on aperture radius, with best values near 2.5-3.0 pixels. We adopt a radius of 3.0 pixels for all of our photometry.

The aperture photometry for CoRoT- 1 at $3.6 \mu \mathrm{m}$, uncorrected for instrument systematic effects, is shown in the top panel of Figure 1. The corresponding time series at $4.5 \mu \mathrm{m}$ is shown in the top panel of Figure 2.

\subsection{CoRoT-2}

CoRoT-2 $(V=12.6, K=10.3)$ observations at $3.6 \mu \mathrm{m}$ began on 2009 November 24 at 18:22 UT (orbital phase 0.4), for a duration of 467.6 minutes. CoRoT-2 being brighter than CoRoT-1, these observations used subarray mode. We collected 215 data cubes, each comprising $642 \mathrm{~s}$ exposures of $32 \times 32$ pixels, followed by three data cubes of blank sky.

We perform photometry on the CoRoT- 2 data cubes in a similar manner to the full-frame data for CoRoT-1. We inspect the aperture photometry for the 64 frames within each data cube, and zero-weight outliers exceeding the average by more than $4 \sigma$. The first frame in each data cube is consistently found to be an outlier, and is always ignored. We analyze the remaining 63-frame data cubes so as to produce two distinct versions of the photometry, and we perform the entire eclipse-fitting and error analysis for each version. In the first (default) version, we average the background-subtracted aperture photometry for all

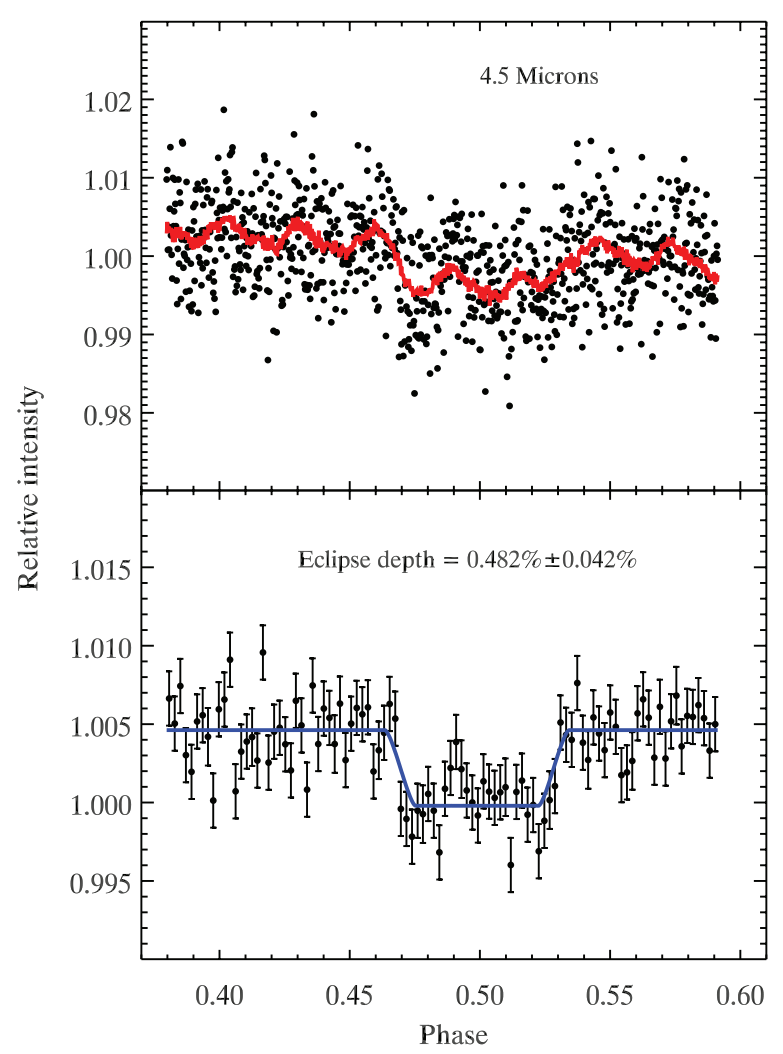

Figure 2. Upper panel: photometry of CoRoT-1 vs. orbital phase, at $4.5 \mu \mathrm{m}$ (points), with the decorrelation function overplotted (red line). Bottom panel: photometry with the decorrelation function removed, and binned to a resolution of approximately 0.002 in orbital phase (100 bins), with the best-fit eclipse curve overlaid (blue line). The error bars are based on the scatter of individual points within each bin. The best-fit central phase is $0.4992 \pm 0.0014$.

(A color version of this figure is available in the online journal.)

63 frames in each data cube, to produce a single photometric point. For the second version, we use each of the 63 frames as a separate photometric point. Using these individual frames potentially exploits the short-term pointing jitter to better define the intra-pixel effect. However, in practice the frame-to-frame fluctuations within a data cube are dominated by photon noise for these relatively faint stars. The eclipse results and errors from these two versions of the photometry are close to being identical (difference much less than $1 \sigma$ ). Note that the default method is essentially just a binning of the data. We prefer the default version because the eclipse plot (Figure 3) is visually clearer.

We also explored a third version of the photometry, wherein we average the actual data frames in each data cube, omitting the first frame and using a median filter to reject outlying pixels. We then perform aperture photometry on the averaged frame. This method gives essentially the same result as our default method: the eclipse amplitude (see below) differed by $0.4 \sigma$ and the phase differed by $1.14 \sigma$.

\subsection{Eclipse Amplitudes}

CoRoT-1 and -2 have well defined transit parameters (planetary and stellar radii, orbit inclination, etc.). We adopt these parameters from Barge et al. (2008) and Alonso et al. (2008), and we calculate eclipse curves numerically, following Todorov et al. (2010). We maintain the known durations of ingress and egress, but we vary the central phase and eclipse depth when fitting to the photometry. 


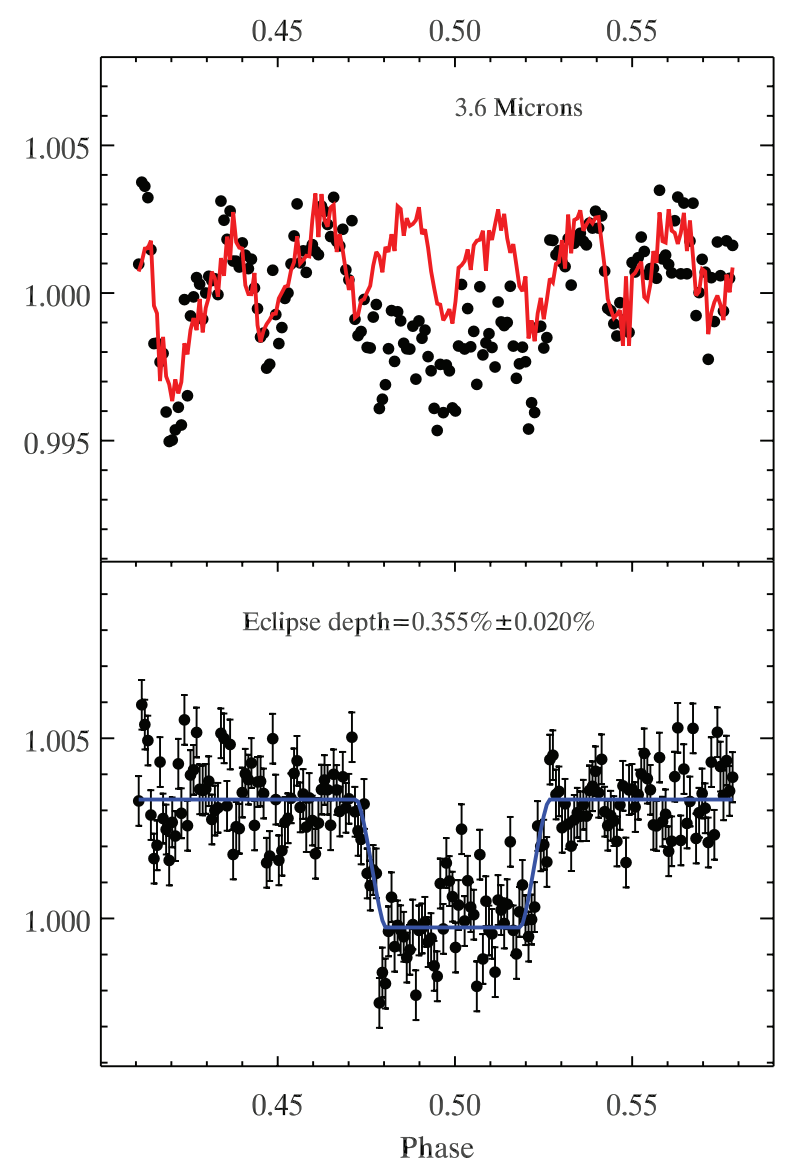

Figure 3. Upper panel: photometry of CoRoT-2 vs. orbital phase, at $3.6 \mu \mathrm{m}$ (points), with the decorrelation function overplotted (red line). Each point is the average of 63 temporal frames in a data cube of $32 \times 32$ pixels times 64 temporal frames (dropping the first). Bottom panel: photometry with the decorrelation function removed, with the best-fit eclipse curve overlaid (blue line). The error bars are the theoretical limit based on the photon and read noise. The best-fit central phase is $0.4994 \pm 0.0007$.

(A color version of this figure is available in the online journal.)

Both the 3.6- and $4.5 \mu \mathrm{m}$ channels show the well-known intra-pixel sensitivity variation (Morales-Calderon et al. 2006). We fit for the eclipse depth and the coefficients of the intrapixel correction using linear regression. The details of the fitting procedure vary with wavelength, but at all wavelengths we search for the best central phase by repeating the linear regressions at many phase values in a dense grid (spacing 0.0002 in phase), and we adopt the central phase yielding the best $\chi^{2}$. We always perform this grid-search in phase when fitting for eclipse amplitude, for both planets at all wavelengths and also for our Monte Carlo trials to define errors (see below).

We apply the linear regressions using an iterative procedure. We first decorrelate the photometry to remove the intra-pixel effect, while ignoring the eclipse, and then we fit for the eclipse depth using a second regression on the decorrelated data. After removing the fitted eclipse depth from the original photometry, we then re-fit and decorrelate the intra-pixel variation, then re-fit the eclipse. This procedure converges in two cycles. In principle, iteration is unnecessary because the regressions are linear and an identical result can be achieved by solving simultaneously for both the intra-pixel coefficients and the eclipse depth. (We verified this by actually doing the simultaneous fit for a simple case.) Nevertheless, we use the iterative procedure because in actual practice it is more flexible and it affords the opportunity to use variants of the fit that would be awkward to implement in a simultaneous solution. This should become apparent from the description below.

At $3.6 \mu \mathrm{m}$ the intra-pixel signature in the photometry $(\sim 2 \%$ peak-to-peak) is larger than the eclipse (see the top panel of Figure 1). Our first step is to solve for a provisional intrapixel decorrelation. The provisional decorrelation function is assumed to be linear in both $\delta X$ and $\delta Y$, which are defined as the change in $X$ - and $Y$-pixel position of the image centroid after removing a trend in $X$ and $Y$ with time. The approximately $1 \mathrm{hr}$ quasi-periodic jitter in position has peak-to-peak amplitude in $\delta X$ and $\delta Y$ of about 0.03 and 0.08 pixels, respectively. The trends (slow drifts) are smaller, about 0.005 pixels in $X$ over the entire data set, and 0.06 pixels in $Y$. The provisional intra-pixel decorrelation function is linear in both $\delta X$ and $\delta Y$, and includes a term linear in time that accounts for both the slow drift in position as well as possible change in detector sensitivity. We solve for the coefficients using linear regression (matrix inversion), and correct the original photometry using this decorrelation function.

Following the provisional intra-pixel decorrelation, we solve for the eclipse depth, again using linear regression. This regression formally allows a linear baseline in time, but that term is effectively accounted for by the intra-pixel decorrelation of the previous step. We remove the fitted eclipse from the original photometry, and begin the second cycle of the iteration. This implements a more sophisticated version of the intra-pixel decorrelation, expressing the decorrelation function as linear in both time and the radial distance of the image from pixel center (called pixel phase). Because there is slow drift of the image toward pixel center by about 0.06 pixels over the duration of the observations, intrinsic spatial variation in the intra-pixel sensitivity (i.e., a change of spatial slope) may be manifest as a change in the decorrelation coefficient of pixel phase. In this particular case (CoRoT-1 at $3.6 \mu \mathrm{m})$, visual inspection of the data indeed suggested a change in the slope of the intrapixel effect. To allow for this change in slope, we divide the decorrelation into two halves, the first half before mid-eclipse and the second half after mid-eclipse. In effect, this is a minimalist implementation of using a quadratic term in the intrapixel fit. Although it is unconventional, we judge it to be the best approach to this particular case. The coefficients of both halves are found via linear regression on the eclipse-removed data. The separate decorrelation functions for the first and second halves of the data can be discerned in the top panel of Figure 1. Note that they are almost continuous at the break near phase 0.5. None of the conclusions of this paper would be different if we restricted the decorrelation to more conventional methodology, but the quality of the $3.6 \mu \mathrm{m}$ eclipse fit for CoRoT-1 would be degraded.

After this decorrelation, we again remove the intra-pixel effect from the original photometry, and re-solve for the final eclipse depth and a possible linear baseline via regression. The eclipse fit uses all of the data, not breaking it into halves. Decorrelated CoRoT-1 data and the best-fit eclipse are shown in the middle panel of Figure 1, and are binned (to 100 bins) in the bottom panel of Figure 1.

We use a nearly identical procedure to fit the $3.6 \mu \mathrm{m}$ eclipse of CoRoT-2, shown in Figure 3, except that we do not break the decorrelation at mid-eclipse. The first $\sim 30$ minutes of these data (not illustrated in Figure 3) exhibit a transient decrease in flux, similar to the ramp effect seen at longer wavelength, but decreasing instead of increasing, and not correlated with 
Table 2

Eclipse Central Times and Phase for CoRoT-1 and CoRoT-2.

\begin{tabular}{clcc}
\hline \hline Planet & Wavelength & HJD & Phase \\
\hline CoRoT-1 & $3.6 \mu \mathrm{m}$ & $2455162.1643 \pm 0.0036$ & $0.5012 \pm 0.0024$ \\
& 4.5 & $2455159.1433 \pm 0.0021$ & $0.4992 \pm 0.0014$ \\
CoRoT-2 & 3.6 & $2455160.4496 \pm 0.0012$ & $0.4994 \pm 0.0007$ \\
& 4.5 & $2454771.7598 \pm 0.0007$ & $0.4976 \pm 0.0004$ \\
& 8.0 & $2454771.7633 \pm 0.0033$ & $0.4992 \pm 0.0019$ \\
\hline
\end{tabular}

Notes. Orbital phase for CoRoT-1 used $T_{0}=2454524.62324$ and $P=$ 1.5089686 days (Gillon et al. 2009a). For CoRoT-2 we used $T_{0}=$ 2454237.53562 (Alonso et al. 2008) and $P=1.7429935$ days (Gillon et al. 2010).

the image position on the detector. Transient effects at this wavelength are not well understood, so we simply omit the 19 data cubes prior to orbital phase 0.41 .

Another difference for CoRoT-2 is that a correction is needed for diffracted light from an M-dwarf lying 4 arcsec distant (Gillon et al. 2010). Since we also re-analyze archival data at 4.5 and $8 \mu \mathrm{m}$ for CoRoT-2 (see below), we need to estimate the diffracted light contributed by the M-dwarf in the CoRoT-2 aperture at $3.6,4.5$, and $8 \mu \mathrm{m}$. We calculated the flux ratio (M-dwarf to CoRoT-2) in the IRAC bands, using the flux estimation tool (STAR-PET) on the Spitzer Web site, and the Two Micron All Sky Survey $K$ magnitudes and $J-K$ colors of the two stars. Knowing their relative brightness, we also need to know the fraction of the M-dwarf flux that is diffracted into the photometry aperture for CoRoT-2. We estimated this by placing the aperture at a symmetric location on the other side of CoRoT-2, where the diffracted light is contributed almost exclusively by CoRoT-2 itself. Using that diffracted fraction together with the flux ratio of M-dwarf to CoRoT-2, we infer that the diffracted light from the M-dwarf contributes 5.9\%, 5.0\%, and $8.3 \%$ to CoRoT-2 at 3.6, 4.5, and $8.0 \mu \mathrm{m}$, respectively. The eclipse photometry and derived parameters for CoRoT- 2 in our figures and tables have all been corrected for this diffracted light. Gillon et al. (2010) inferred $16.4 \%$ and $14.3 \%$ at 4.5 and $8 \mu \mathrm{m}$, respectively, but he used aperture radii of 4.0 and 3.5 pixels, respectively, versus 3.0 pixels in our case.

As a check, we repeated our diffracted light correction using apertures having the same size as Gillon et al. (2010). Because the diffracted light is not uniform, the values do not simply scale as the area of the aperture. For the same apertures as Gillon et al. (2010), we obtain corrections of $14.2 \%$ and $12.0 \%$ at 4.5 and $8.0 \mu \mathrm{m}$, respectively, in reasonably good agreement with the independent determination of Gillon et al. (2010). Uncertainty in the diffracted light correction is not included in our eclipse amplitude error estimates. Given our good agreement with the diffracted light corrections of Gillon et al. (2010), and given that we use smaller photometric apertures than Gillon et al. (2010), we conclude that uncertainty in the diffracted light correction does not contribute significantly to the errors on our measured eclipse depths.

The best-fit eclipse depths and errors are listed in Table 1, and the central phases and errors are listed in Table 2.

\subsection{Error Estimation}

The ideal method to calculate errors would be to repeat all of the observations and analysis, and compare the results from analyzing many independent sets of observations. This is obviously impractical, so we mimic some key aspects of that ideal procedure. We generate fake photometric data sets having the same properties as the real photometry, and we repeat the entire iterative fitting process-including intra-pixel corrections and ramp fitting - on each fake data set. We calculate the standard deviation of the collection of eclipse depths and central phases resulting from the repetitions of the analysis on the fake data.

To generate each fake data set, we subtract the best-fit eclipse curve (plus baseline and intra-pixel decorrelation function) from the original photometry to produce a set of photometric residuals. We likewise produce a set of image position residuals by subtracting a multi-point running average of the $X$ - and $Y$-pixel positions from each individual $(X, Y)$ position measurement. We permute all of the residuals and add them back to the best-fit function (photometry) or running average coordinate (position) to make an individual fake data set. We permute the residuals using two methods, to make two distinct collections of fake data. The first permutation method scrambles the residuals randomly, which is equivalent to the conventional bootstrap Monte Carlo technique (Press et al. 1992). We generate $10^{4}$ bootstrap data sets (trials) using this method, and calculate the standard deviation of eclipse depth and central phase from the distributions of these parameters over the $10^{4}$ trials. These distributions are close to Gaussian.

A second method to permute the residuals preserves their relative order but shifts their initial phase; this is sometimes called the "prayer-bead" method (Gillon et al. 2009b). In this case, the number of trials equals the number of original photometry points. This is 888 for CoRoT-1, and 13,545 for version 2 of the CoRoT-2 subarray photometry. These are adequate to define the distributions of eclipse depth and phase. The prayer-bead method is more sensitive to the presence of red noise in the data. Nevertheless, we find that the distribution of eclipse depth remains consistent with a Gaussian, but for CoRoT-1 the distribution of eclipse phase shows that about $7 \%$ of the central phases lie below the $3 \sigma$ point in the distribution. We attribute this to the presence of some red noise before mideclipse, visible in the bottom panel of Figure 1.

For CoRoT-2, the distributions of eclipse depth and phase were close to Gaussian, so errors from the prayer-bead method were quite close to the values from the bootstrap method. This indicates relatively little red noise in the CoRoT-2 data (after we omitted the first 19 data points, as noted above). For both CoRoT-1 and -2, we adopted the greater of the bootstrap and prayer-bead errors for each parameter. CoRoT-1 errors are uniformly larger than for CoRoT-2 because the star is fainter and the red noise is slightly greater. Tables 1 and 2 list the errors on eclipse depth and central phase for all three eclipses, plus our results from re-analysis of CoRoT-2 at 4.5 and $8 \mu \mathrm{m}$ (see below).

\subsection{CoRoT-2 at 4.5 and $8 \mu \mathrm{m}$}

We check our methodology by analyzing archival Spitzer data for CoRoT-2 at 4.5 and $8 \mu \mathrm{m}$, for comparison to the results of Gillon et al. (2010). Our analysis at $4.5 \mu \mathrm{m}$ proceeds as described above for CoRoT-1. At $8 \mu \mathrm{m}$ our eclipse fitting procedure uses a "ramp" baseline (Deming et al. 2006; Knutson et al. 2009) that is fit simultaneously with the eclipse depth by linear regression. The ramp is comprised of a term linear in time, a term linear in the logarithm of time, with a zero point on the time axis as described by Todorov et al. (2010). We also find that the photometry exhibits a rather rapid decrease in flux during the first 100 data points. Investigating this, we find an approximately 0.1 pixel change in the image $Y$-position during those first 100 points. This transient positional drift is not in sync 
with the well-known telescope pointing oscillation. Although the pointing oscillation has not (to our knowledge) been shown to affect $8 \mu \mathrm{m}$ Spitzer photometry, the 0.1 pixel transient drift apparently does. We therefore include a $Y$-position term in the linear regression fit for the eclipse depth. Without this term, the eclipse depth would be $0.42 \%$, versus our result of $0.446 \%$ (Table 1).

We also perform trial fits using the double-exponential ramp of Agol et al. (2010). These fits, like the log ramp discussed above, omit the first 100 points and include a $Y$-position term. The ramp observed in the $8 \mu \mathrm{m}$ data (illustrated by Gillon et al. 2010) is very shallow, and the scatter is relatively large compared to the ramp-related flux change. For this reason, we use a single exponential ramp, not a double exponential ramp. We experimented with double-exponential fits, but our Levenberg-Marquardt fitting procedure produced degeneracies when attempting to fit two exponentials to such a shallow ramp. We believe that only one exponential is warranted in this case. Moreover, the best-fit exponential ramp is close to a straight line, since the ramp curvature is minimal. As will become apparent in Section 3.2, the $8 \mu \mathrm{m}$ eclipse depth of CoRoT-2 is crucial to the interpretation of our results, so we will return to the implications of fitting the exponential ramp during that discussion.

Our results for CoRoT-2 at 4.5 and $8 \mu \mathrm{m}$ are included in Tables 1 and 2 . The eclipse depth using the exponential ramp at $8 \mu \mathrm{m}$ is included in Table 1 , but the phase results for that ramp are the same as the log ramp, and are not listed separately in Table 2. Overall, we find excellent agreement with Gillon et al. (2010).

\section{RESULTS AND DISCUSSION}

\subsection{Orbital Phase}

For CoRoT-1, we compute the weighted average of the central eclipse phase using both 3.6 and $4.5 \mu \mathrm{m}$ eclipses, adopting weights equal to the inverse of the variance of each measurement. This yields a central phase of $0.4994 \pm 0.0013$, and $|e \cos (\omega)|<0.006$ to $3 \sigma$. Our limit indicates that the orbit is close to circular, but a small non-zero eccentricity (such as we infer for CoRoT-2, see below) is not excluded.

For CoRoT-2, Gillon et al. (2010) found $e \cos (\omega)=$ $-0.00291 \pm 0.00063$. Our result for the $3.6 \mu \mathrm{m}$ eclipse (central phase at $0.4994 \pm 0.0007$ ) is displaced in the same direction as Gillon et al. (2010) infer, but with insufficient precision to confirm or reject the Gillon et al. (2010) claim. Combining our $3.6 \mu \mathrm{m}$ result with the eclipses analyzed by Gillon et al. (2010) could increase the significance of the total result. For maximum consistency, we re-analyzed the 4.5 and $8 \mu \mathrm{m}$ eclipse data, as described above. We verified that our adopted transit ephemeris (see Table 2) should not be a significant source of error when propagated to the eclipse times. Weighting each eclipse phase (3.6, 4.5, and 8, see Table 2) by the inverse of its variance yields an average central phase of $0.49809 \pm 0.00028$. Including the $28 \mathrm{~s}$ for light to cross the planetary orbit, we expect to find the eclipse at phase 0.500019 if the orbit is circular. Hence, we derive $e \cos (\omega)=-0.0030 \pm 0.0004$. The excellent agreement with Gillon et al. (2010) is in part because we are analyzing much of the same data. However, the result is heavily weighted by the single eclipse at $4.5 \mu \mathrm{m}$, which is a reason to be cautious concerning a claim of non-zero eccentricity. Nevertheless, at face value we are able to reproduce the result of Gillon et al. (2010) using an independent analysis, and improve the precision slightly. Gillon et al. (2010) point out that a non-zero eccentricity

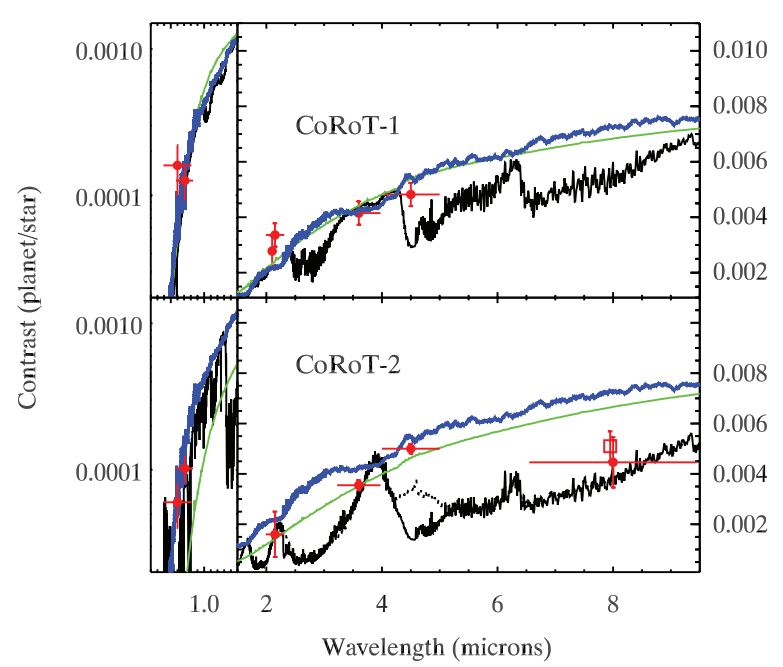

Figure 4. Planet to star contrast ratios for CoRoT-1 and CoRoT-2 vs. wavelength, from Table 1. The short wavelength data are on left panels (contrast on log scale) and longer wavelength data on the right (contrast on linear scale). Data from CoRoT, ground-based at $2 \mu \mathrm{m}$, and Spitzer are all plotted with red points. Error bars on the abscissa give the half-intensity wavelength limits of the bandpasses. For CoRoT-2 we plot our re-analysis of the Gillon et al. (2010) data at 4.5 and $8 \mu \mathrm{m}$, but the original Gillon et al. (2010) values are similar. The square point at $8.0 \mu \mathrm{m}$ is the eclipse depth using the exponential ramp (see Table 1). The black curves are non-inverted Burrows models having 30\% redistribution of stellar irradiance to the night side, with no extra absorbing layers at high altitude. For CoRoT-2, the black dotted portion near $4.5 \mu \mathrm{m}$ is the same Burrows model, only lacking $\mathrm{CO}$ absorption. The blue lines are inverted models from Fortney and collaborators (Fortney et al. 2005, 2006, 2008) having TiO absorption, and no re-distribution of stellar irradiance. The green lines are blackbodies having $T=2460 \mathrm{~K}$ (CoRoT-1, Rogers et al. 2009) and $T=1866 \mathrm{~K}$ (CoRoT-2, Cowan $\&$ Agol 2010). The reduced $\chi^{2}$ values for the CoRoT-1 data as compared to the different models are: conventional model (black line) $=12.6$, inverted model (blue line $)=2.4$, blackbody $($ green line $)=1.9$. For CoRoT-2, the reduced $\chi^{2}$ values for those models are 61.4, 30.4, and 12.5, respectively. (These values use the log ramp point at $8 \mu \mathrm{m}$, not the exponential ramp.) The reduced $\chi^{2}$ value for CoRoT-2 compared to the non-inverted model without $\mathrm{CO}$ absorption (dotted portion) is 13.5 .

(A color version of this figure is available in the online journal.)

does not require an additional planet in the system, since incomplete two-body tidal circularization is a plausible alternative for this system.

\subsection{Atmospheric Temperature Structure}

Our results for both planets are summarized in Figure 4, which shows all available eclipse data in comparison to various models. The caption of Figure 4 gives reduced $\chi^{2}$ values for the comparison between each model and the eclipse data. Since Figure 4 is a comparison of the data to model predictions, not a fit involving adjustable parameters, we take the degrees of freedom to equal the number of data points when calculating the reduced $\chi^{2}$.

The model comparison for CoRoT-1 (top panels of Figure 4) suggests an inverted atmospheric temperature structure. The best overall account of the data is actually produced using a $T=2460 \mathrm{~K}$ blackbody spectrum (Rogers et al. 2009, green line, see reduced $\chi^{2}$ values in the caption of Figure 4). However, this likely indicates the presence of a high altitude absorbing layer, and such layers are implicated in driving the inversion phenomenon (Burrows et al. 2007; Knutson et al. 2008). The nature of the absorber is the subject of current debate (Fortney et al. 2008; Spiegel et al. 2009). The conventional model (black line, Burrows et al. 2008) shows significant absorption due to the CO bandhead that occurs near $4.7 \mu \mathrm{m}$, and the Spitzer data 
show no sign of being affected by this feature. An inverted model using $\mathrm{TiO}$ absorption (blue line) shows much better agreement with the data than the non-inverted model, but does not account particularly well for the ground-based $(2 \mu \mathrm{m})$ measurements. An atmosphere with a nearly isothermal region over extended heights will produce a blackbody-like spectrum, and can be regarded as a special case of an inverted temperature structure. The inverted and blackbody models for CoRoT-1 both give good agreement with the Spitzer data, as well as the CoRoT optical eclipse measurements (Snellen et al. 2009; Alonso et al. 2009b). This indicates that the optical emission is predominately thermal in origin. The models that account for our Spitzer data, when compared to the optical eclipses (Figure 4), leave little room for a reflected light component. Based on the models of Seager et al. (2000), a geometric albedo near unity would produce a reflected light eclipse depth of approximately 520 ppm, whereas the difference between the CoRoT-1 observations (Snellen et al. 2009) and the inverted model (blue curve in Figure 4) is 84 and $21 \mathrm{ppm}$ at 0.6 and $0.71 \mu \mathrm{m}$, respectively. Also, Cowan \& Agol (2010) inferred a Bond albedo of $<10 \%$ for CoRoT-1. Our results therefore support the conclusion of Snellen et al. (2009) and Cowan \& Agol (2010) that CoRoT-1 is a dark planet.

CoRoT-2 (bottom panels of Figure 4) is more complex than CoRoT-1. A conventional model (black line, Burrows et al. 2008) produces excellent agreement with all of the data except for the $4.5 \mu \mathrm{m}$ point, where the disagreement is substantial. Since the $4.5-3.6 \mu \mathrm{m}$ contrast ratio is even greater than for CoRoT-1, a temperature inversion is suggested. But inverted models do not reproduce the $8 \mu \mathrm{m}$ contrast and, based on the reduced $\chi^{2}$ values (Figure 4 caption), no model gives a reasonable account of the total data. Both the 4.5 and $8 \mu \mathrm{m}$ observed values are in good agreement between our analysis and Gillon et al. (2010), so the problem does not seem to lie with the observations. We first mention some caveats, and then we suggest two hypotheses to account for the contrast values of this unusual planet.

One caveat that applies to CoRoT- 2 is the fact that the star is active (Alonso et al. 2008). However, because the planet passes behind the star during eclipse, there is no time-variable blocking of active regions on the stellar disk. The primary consequence of stellar activity is the photometric variation of the star itself. This variation can manifest itself in two ways. First, stellar variations can appear directly in the eclipse curve. The dominant stellar variation will be due to rotational modulation of active regions, with a 4.5 day period (Lanza et al. 2009). This timescale is more than an order of magnitude longer than the $2.2 \mathrm{hr}$ eclipse duration. Although rotation of active regions can still affect eclipse data (e.g., by perturbing the photometric baseline), we do not discern any indications of it, so we interpret our data at face value. The second way in which stellar variations can affect eclipse depth is through the normalization. When the star is fainter, the disappearance of the planet during eclipse translates to a larger fraction of the stellar flux. This effect can alter eclipse depths on long timescales. However, the 4.5 and $8 \mu \mathrm{m}$ observations made by Gillon et al. (2010) were simultaneous, so long-term stellar variability cannot be a factor in the puzzling spectrum of CoRoT-2.

A final caveat concerns the ramp effect for CoRoT-2 at $8 \mu \mathrm{m}$. We find that fitting the exponential model of Agol et al. (2010) increases the eclipse depth to $0.51 \%$ (Table 1 ). However, this does not alter the situation concerning the interpretation of the CoRoT-2 results, so we now discuss two hypotheses to account for the totality of the CoRoT-2 data as summarized in Tables 1 and 2 .

\subsection{Possible Mass Loss for CoRoT-2}

Our first hypothesis for CoRoT-2 is that the planetary atmosphere is well described by a conventional (non-inverted) model, but the $4.5 \mu \mathrm{m}$ eclipse appears anomalously deep because it contains carbon monoxide emission lines due to mass loss. We find that a conventional model lacking $\mathrm{CO}$ absorption (see Figure 4) does not increase the contrast sufficiently in the $4.5 \mu \mathrm{m}$ band to account for the data-the reduced $\chi^{2}$ is 13.5 (Figure 4). Actual emission from mass loss would be required. Mass loss for close-in giant exoplanets can occur via tidal stripping (Li et al. 2010), and also via energy deposition from stellar UV flux. The latter process is particularly important for planets orbiting young, UV-bright stars (Baraffe et al. 2004; Hubbard et al. 2007). CoRoT-2 orbits very close-in, where the tidal force is strong (0.026 AU, Barge et al. 2008). Moreover, the star is young and active (Bouchy et al. 2008), possibly as young as 30 Ma (Guillot \& Havel 2010). Hence, both mass-loss mechanisms are potentially important for this planet.

$\mathrm{Li}$ et al. (2010) have predicted significant $\mathrm{CO}$ emission in the $\Delta V=2$ overtone bands near $2.29 \mu \mathrm{m}$, due to tidally stripped mass loss from WASP-12. This mass should also emit in the $\mathrm{CO} \Delta V=1$ bands, which are intrinsically stronger than the overtone bands, and arise from upper levels that are easier to excite. Emission from the $\Delta V=1$ bands will fall within the $4.5 \mu \mathrm{m}$ bandpass, increasing the eclipse depth. Tidal-induced mass loss is at least qualitatively consistent with the apparent non-zero eccentricity of the orbit. However, recent results show that the orbit of WASP-12b is likely to be more circular than Li et al. (2010) suppose (Campo et al. 2010; Husnoo et al. 2010). The evidence for non-circularity is better in the CoRoT-2 case than for WASP-12, so we explore whether a mass-loss and CO emission scenario might be profitably applied to CoRoT-2.

We calculate what mass-loss rate is required to increase the $4.5 \mu \mathrm{m}$ contrast sufficiently over the conventional model to account for the observed eclipse depth. We compare the requisite mass-loss rate with model calculations for both tidal-stripping, and evaporation by stellar UV flux. If the required mass-loss rate is (for example) so large that the planet would disappear within an unacceptably small timescale, then we could discard the mass-loss hypothesis.

Prior to calculating the mass loss required to account for the $4.5 \mu \mathrm{m}$ eclipse, we mention a potentially serious problem with this hypothesis. This problem derives from the eclipse curve itself. In a variant of our bootstrap error analysis, we allowed the ingress and egress times of the eclipse to vary. We implemented variations in ingress/egress time by applying linear transformations to the time axis prior to second contact, and subsequent to third contact. We find that the $1 \sigma$ precision of the observed ingress/egress time is about $10 \%$. This implies that the radius of any $\mathrm{CO}$-emitting volume cannot be more than about $30 \%$ larger $(3 \sigma$ limit $)$ than the radius of the planet. Given the requisite mass-loss rate (see below), we calculated a synthetic spectrum for the resultant CO column density of $10^{19} \mathrm{~cm}^{-2}$, adopting excitation temperatures from $3000 \mathrm{~K}$ to $15,000 \mathrm{~K}$. Many individual lines in this spectrum are optically thick, and attain intensities closely equal to the Planck function at the excitation temperature. However, the line density in the $4.5 \mu \mathrm{m}$ Spitzer bandpass is insufficient to produce the required eclipse flux unless the excitation temperature exceeds 15,000 K. Since $\mathrm{CO}$ is primarily dissociated at such temperatures, we cannot 
easily match the required eclipse flux using such a compact source of CO emission. Nevertheless, the details of mass loss in the Roche lobe and through the inner Lagrangian point are not completely understood, so we present our calculation of the mass loss required to account for the $4.5 \mu \mathrm{m}$ eclipse depth.

Let the continuum flux from the star, integrated over the $4.5 \mu \mathrm{m}$ band, be denoted $F_{s}$, in erg $\mathrm{cm}^{-2} \mathrm{~s}^{-1}$. Let the flux from the hypothetical $\mathrm{CO}$ cloud be denoted $F_{\mathrm{CO}}$ in the same units. Then, the excess over the standard model atmosphere for the planet (Figure 4) requires

$$
F_{\mathrm{CO}} \approx 0.005 F_{s} .
$$

A Phoenix model atmosphere for the star (Hauschildt et al. 1999), integrated over the $4.5 \mu \mathrm{m}$ bandpass, gives the same flux as blackbody having $T=5237 \mathrm{~K}$, so $F_{s}=\Delta \nu \Omega_{s} B_{v}$, where $B_{v}=5.17 \times 10^{-6}$ is the Planck function (in cgs units) at $5237 \mathrm{~K}, \Omega_{s}$ is the solid angle of the star as seen from Spitzer, and $\Delta v$ is the bandwidth of the $4.5 \mu \mathrm{m}$ band in $\mathrm{Hz}$. We also have $F_{\mathrm{CO}}=L /\left(4 \pi d^{2}\right)$, where $L$ is the luminosity of the CO-emitting cloud within the $4.5 \mu \mathrm{m}$ band (erg s${ }^{-1}$ ), and $d$ is the distance to the system. The solid angle $\Omega_{s}=\pi R^{2} / d^{2}$, where $R$ is the radius of the star. We substitute for $d^{2}$ in the expression for $F_{\mathrm{CO}}$, and then (1) becomes

$$
L \approx 0.2 \Delta \nu B_{v} R^{2} \approx 6.2 \times 10^{28} \mathrm{erg} \mathrm{s}^{-1} .
$$

The number of $\mathrm{CO}$ molecules required to produce this luminosity depends on their excitation state and on the Einstein-A values for the emission. We first adopt a thermal distribution at $T=3000 \mathrm{~K}$ for the $\mathrm{CO}$ vibrational levels, and we use the rotationless Einstein A-values $A_{j i}$ for $\Delta V=1$ from Okada et al. (2002). Summing over the vibrational levels, we find that the effective emitting rate is $28 \mathrm{~s}^{-1}$ per $\mathrm{CO}$ molecule. Since $h v \approx 4.42 \times 10^{-13}, L \approx 1.4 \times 10^{41}$ photons $\mathrm{s}^{-1}$. This requires $4.9 \times 10^{39} \mathrm{CO}$ molecules in the emitting volume. Adopting a solar carbon abundance $\left(10^{-3.5}\right)$, and stipulating that all of the carbon appears in $\mathrm{CO}$, the total mass in the emitting volume is approximately $1.5 \times 10^{-11}$ Jupiters.

To determine a mass-loss rate from the total mass in the emitting volume, we must estimate the transit time of $\mathrm{CO}$ molecules. This has been discussed by Li et al. (2010), who conclude that mass flows through the Roche lobe at the sound speed, and forms a disk around the star. Most of that disk emission will not be modulated by the secondary eclipse, so our observations refer only to the mass flowing out of the Roche lobe itself. The relevant time is therefore the Roche lobe radius $a\left(M_{p} / 3 M_{s}\right)^{1 / 3}$ divided by the sound speed $(\gamma P / \rho)^{1 / 2}$. We calculate a Roche lobe radius for CoRoT-2 of $4.3 \times 10^{5} \mathrm{~km}$, and a sound speed of $4.5 \mathrm{~km} \mathrm{~s}^{-1}$. These values yield a mass-loss rate of $\sim 5 \times 10^{-9} M_{J}$ per year. This value is in close accord with a minimum value for WASP-12, calculated by Lai et al. (2010). It is also a reasonable value for a giant planet close-in to a young active star (Hubbard et al. 2007).

The greatest uncertainty in the above calculation is the excitation state of the $\mathrm{CO}$ molecules. Because the population of the vibrational levels varies exponentially with vibrational temperature, the effective emitting rate could vary by orders of magnitude and still be consistent with our ignorance. If $\mathrm{CO}$ lost from the planet is vibrationally cold $(T=300 \mathrm{~K}$, for example), as will tend to happen in the absence of collisional excitation, then the effective emission rate drops by over four orders of magnitude, and the required mass-loss rate increases by that factor, and becomes unacceptably large. Indeed, in the arguably applicable limit of no collisional excitation, each CO molecule would emit approximately one photon as it expanded from the planetary atmosphere through the Roche lobe. That limit would require a mass-loss rate as high as $10^{-2} M_{J}$ per year, which is unacceptably high.

Although the requisite mass-loss rate is within the range for tidal-stripping and UV-energy deposition models, we conclude that this CO-emission hypothesis is an unlikely interpretation of the Spitzer data, due to the difficulty with the ingress/egress time and the necessity of maintaining collisional excitation. However, it cannot be absolutely ruled out without more detailed models as well as observed high resolution spectroscopy of the system. If this hypothesis could be confirmed, the consequent lack of an atmospheric temperature inversion for this planet-orbiting an active star-would be consistent with the emerging anticorrelation between the presence of inversions and stellar activity levels (Knutson et al. 2010).

\subsection{An Inverted Atmosphere Variant for CoRoT-2}

A second hypothesis to account for CoRoT-2b is a variant of an inverted atmospheric structure. The $8 \mu \mathrm{m}$ radiation may hypothetically emerge from deeper and cooler atmospheric layers, whereas the shorter wavelengths are formed in a high altitude layer that is heated by absorption. Absorption in a high altitude layer has been implicated (Burrows et al. 2007) as driving atmospheric temperature inversions, by absorbing stellar irradiance and heating the planetary atmosphere at altitude. Radiative equilibrium of a high altitude absorbing layer that is optically thick in the optical and near-IR could potentially shield lower levels of the atmosphere from radiative heating. A high altitude layer would re-emit both to space and to lower levels of the atmosphere, but the net downward flux would be reduced by upward emission to space. If the opacity of the absorbing layer is high in the optical and near-IR $(\lambda<5 \mu \mathrm{m})$, eclipse observations at those wavelengths may sense only the absorbing layer, whereas longer wavelengths (e.g., $8 \mu \mathrm{m}$ ) may penetrate and sense the cooler lower atmosphere.

Recently, Guillot \& Havel (2010) have concluded that the IR opacity of CoRoT-2's atmosphere is greater than normal. We are here hypothesizing exactly the opposite of that conclusion, but based in part on the additional $3.6 \mu \mathrm{m}$ eclipse result that was not available to Guillot \& Havel (2010).

One immediate problem with this hypothesis is that $8 \mu \mathrm{m}$ radiation is not believed to be formed any deeper than the shorter wavelength IRAC bands (Burrows et al. 2007). Hence, some additional source of short wavelength opacity is required. Scattering by micron-sized haze particles or aerosols is a potential source of the required opacity if such particles can be lofted and maintained at high altitudes. Haze due to smaller particles at high altitudes has been inferred for other planets (Pont et al. 2008). However, several caveats should be cited with regard to this hypothesis. First, most scattering opacities from small particles have a very broad dependence on wavelength, whereas a sharper long-wavelength cutoff might be required. If the extra opacity is from absorption (as opposed to scattering), then it might perturb the atmospheric temperature gradient so that the cooler lower atmosphere we envision might not exist.

This hypothesis of a heated high altitude layer and a cooler lower atmosphere brings to mind the situation with respect to the global energy budget of HD 189733b. Barman (2008) pointed out that the efficiency of zonal heat redistribution can be highly depth dependent. Deeper layers can redistribute heat more efficiently because their radiative time constant (Iro \& Deming 
2010) is comparable to or exceeds the time for advection of heat by zonal winds. In that case the lower atmosphere responds primarily to the day-night average irradiation, whereas the upper atmosphere comes to radiative equilibrium with day-side irradiation on a short timescale. If $8 \mu \mathrm{m}$ radiation from CoRoT-2 arises from deeper layers, then this effect can in principle act to reinforce the presence of a temperature inversion.

If this second hypothesis is correct, then high opacity at optical and near-IR wavelengths could produce a blackbody spectrum at these wavelengths. An $1866 \mathrm{~K}$ blackbody (green line on Figure 4) produces a reasonable agreement with the 3.6 and $4.5 \mu \mathrm{m}$ data, but is below the optical CoRoT measurements (Alonso et al. 2009a; Snellen et al. 2010). Cowan \& Agol (2010) invoked a simple analytic model of the published photometric observations of close-in exoplanets, and inferred $T=1866 \mathrm{~K}$ and a Bond albedo of $16 \% \pm 7 \%$ for CoRoT-2b. This is qualitatively consistent with our second hypothesis for this planet. Ground-based JHK eclipse measurements of this unusual planet would be very useful in defining the blackbody shape and temperature of the near-IR spectrum.

This work is based on observations made with the Spitzer Space Telescope, which is operated by the Jet Propulsion Laboratory, California Institute of Technology, under a contract with NASA. Support for this work was provided by NASA. H.K. is supported by a fellowship from the Miller Institute for Basic Research in Science. E.A. acknowledges support under NSF CAREER grant no. 0645416. A.B. was supported by NASA grant NNX07AG80G and under JPL/Spitzer Agreements 1328092,1348668 , and 1312647 . He is also pleased to note that part of this work was performed while in residence at the Kavli Institute for Theoretical Physics, funded by the NSF through grant no. PHY05-51164. We thank Dr. Rory Barnes for informative conversations regarding the tidal evolution of CoRoT-2, and an anonymous referee for a very thorough review that improved this paper significantly.

\section{APPENDIX}

Because our results are among the first for exoplanets using Warm Spitzer, we take this opportunity to comment on the photometric quality of the Warm mission exoplanet data. The loss of cryogen has increased the operating temperature of the InSb detectors from $15 \mathrm{~K}$ (cryogenic) to $29 \mathrm{~K}$ (warm), and that has altered some characteristics of the detectors. For example, the "column pull down" effect has become more prominent. Bright stars cause the signal levels to drop for all pixels in the column they overlap.

None of our target stars happen to lie on columns that are noticeably affected by pull-down. Our photometry code calculates the theoretical limiting signal-to-noise ratio based on the Poisson statistics of the total number of electrons recorded from the star, and we include a read noise of 10 electrons for each pixel within the numerical aperture. After fitting the photometric time series to remove the intra-pixel variations and the eclipse, we calculate the scatter of the residuals and compare this to the theoretical limiting noise. For CoRoT-1 at 3.6 and $4.5 \mu \mathrm{m}$, we achieve $87 \%$ and $92 \%$ of the theoretical signalto-noise ratio, respectively. However, this seemingly excellent performance may be misleading because these are relatively faint stars, where the stellar photon noise is high and will tend to dominate instrumental noise. A more sensitive test for possible instrumental red noise is to calculate the reduced $\chi^{2}$ of the binned data, after removing the best-fit eclipse (bottom panels of Figures 1-3). We base the predicted error of each bin (error bars on the figures) on the observed scatter of the unbinned points, reduced by the square-root of the number of points in each bin (typically, 9). On this basis, the reduced $\chi^{2}$ values are 1.10 and 1.31 for CoRoT-1 at 3.6 and $4.5 \mu \mathrm{m}$, respectively. This indicates that a small amount of red noise occurs for timescales longer than about 5 minutes.

In the case of CoRoT-2, the only binning we used was the averaging over 64 frames in each data cube. Measuring the observed scatter after removing the fit, we find a ratio of $83 \%$ when using all individual frames of each 63-frame data cube, but this reduces to $75 \%$ of the theoretical signal-to-noise ratio when we average the frames in each data cube before fitting the eclipse. Like CoRoT-1, this indicates the presence of a small amount of red noise.

We are interested in whether the column pull-down effect causes enhanced noise for stars that lie on affected columns. Unfortunately, there are no suitably bright stars that overlie pulled-down columns in our CoRoT data, nor did we find any optimal test stars in several other Warm Spitzer data sets that we examined. The best test star we located was HD 189314, lying in the Kepler field (D. Charbonneau, PID 60028). This relatively bright star $(K=9.3)$ is above the $1 \%$ nonlinearity limit for the $12 \mathrm{~s}$ exposures we examined. Because pointing jitter moves the star toward and away from pixel center, it modulates the nonlinearity effect simultaneously with the intrapixel effect. We were unable to effectively decorrelate these mixed instrumental effects. However, we were able to evaluate the point-to-point scatter in the photometry by removing a smoothing function (high-pass filtering). We find that the point-to-point scatter in the photometry achieves $76 \%$ of the theoretical signal-to-noise ratio. We tentatively conclude that the column pull-down effect does not add short-term noise to Warm Spitzer photometry, even for stars overlying affected columns. We are unable to evaluate whether it causes increased red noise, but we anticipate that this will become clear as additional Warm Spitzer observations are accumulated.

Finally, we draw attention to another important difference between the cryogenic and Warm missions. With cryogenic data, we sometimes evaluated the background for subarray photometry by considering a median over all pixels in a data cube (fitting to a distribution), and using this single best-fit background value for each of the 64 frames in the data cube. This had the advantage that the larger number of pixels over the entire data cube resulted in a more precisely determined value, but it was premised on the background being constant within each data cube. We find that this premise is no longer accurate for the Warm mission: the background value varies significantly from frame to frame within a subarray data cube. (The background is probably not due to impinging IR radiation, but is more likely to be electronic in nature.) The statistical penalty of having fewer pixels available when measuring the background in individual frames is offset by the necessity of following these frame-to-frame variations.

The background variations are illustrated in Figure 5, where we show the $3.6 \mu \mathrm{m}$ background per frame as a function of the frame number within a data cube, and compare the cryogenic mission (represented by HD 189733) to the Warm mission (represented by CoRoT-2). Note that the 58th frame continues to exhibit a higher background value in the Warm mission, as it did in the cryogenic mission (Harrington et al. 2007; Agol et al. 2010). We find, in agreement with Agol et al. (2010), 


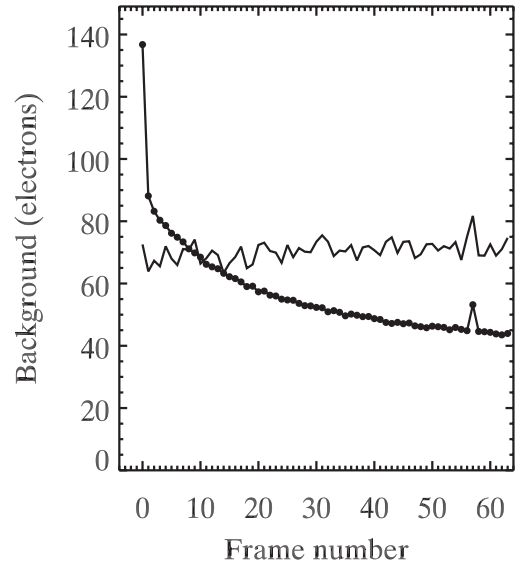

Figure 5. Number of electrons per pixel in the background of CoRoT-2 at $3.6 \mu \mathrm{m}$ (points with line connecting) shown as a function of the frame number in each 64-frame subarray data cube observed using Warm Spitzer. These results are averaged over all 215 data cubes that were acquired, and the exposure time per frame was $2 \mathrm{~s}$. The line without points shows the background for subarray photometry of HD 189733, using observations acquired during the cryogenic mission (Charbonneau et al. 2008). Since background contains both real IR radiation and electronic effects, it is not proportional to exposure time. The shortexposure $(0.1$ s) HD 189733 observations were scaled upward by an arbitrary factor for this plot.

that the photometry from the 58th frame is well behaved if the higher background is accounted for. Because the Warm mission will inevitably observe fainter exoplanet host stars than during the cryogenic mission, accurate background subtraction becomes a high priority. Our $3.6 \mu \mathrm{m}$ photometry for CoRoT-2 used the "per-frame" method that we now find to be necessary, and achieved the $83 \%$ of theoretical signal-to-noise ratio as described above.

Facilities: Spitzer

\section{REFERENCES}

Agol, E., Cowan, N. B., Knutson, H. A., Deming, D., Steffen, J. H., Henry, G. W., \& Charbonneau, D. 2010, ApJ, 721, 1861

Alonso, R., Deeg, H. J., Kabath, P., \& Rabus, M. 2010, AJ, 139, 1481

Alonso, R., Guillot, T., Mazeh, T., Aigrain, S., Barge, P., Hatzes, A., \& Pont, F. 2009a, A\&A, 501, L23

Alonso, R., et al. 2008, A\&A, 482, L21

Alonso, R., et al. 2009b, A\&A, 506, 353

Baraffe, I., Selsis, F., Chabrier, G., Barman, T. S., Allard, F., Hauschildt, P. H., \& Lammer, H. 2004, A\&A, 419, L13

Barge, P., et al. 2008, A\&A, 482, L17

Barman, T. 2008, ApJ, 676, L61

Bouchy, F., et al. 2008, A\&A, 482, L25

Burrows, A., Budaj, J., \& Hubeny, I. 2008, ApJ, 678, 1436

Burrows, A., Hubeny, I., Budaj, J., Knutson, H. A., \& Charbonneau, D. 2007, ApJ, 668, L171

Campo, C. J., et al. 2010, ApJ, in press (arXiv:1003.2763)
Charbonneau, D., Knutson, H. A., Barman, T., Allen, L. E., Mayor, M., Megeath, S. T., Queloz, D., \& Udry, S. 2008, ApJ, 686, 1341

Charbonneau, D., et al. 2005, ApJ, 626, 523

Christiansen, J., et al. 2010, ApJ, 710, 97

Cowan, N., \& Agol, E. 2010, ApJ, in press (arXiv:1001.0012)

Deming, D., Agol, E., Charbonneau, D., Cowan, N., Knutson, H., \& Marengo, M. 2007, in AIP Conf. Proc. 943, The Science Opportunities for the Warm Spitzer Mission, ed. L. J. Storrie-Lombardi \& N. A. Silbermann (Melville, NY: AIP), 89

Deming, D., Harrington, J., Seager, S., \& Richardson, L. J. 2006, ApJ, 644, 560

Deming, D., Seager, S., Richardson, L. J., \& Harrington, J. 2005, Nature, 434, 740

Fazio, G. G., et al. 2004, ApJS, 154, 10

Fortney, J. J., Lodders, K., Marley, M. S., \& Freedman, R. S. 2008, ApJ, 678, 1419

Fortney, J. J., Marley, M. S., Lodders, K., Saumon, D., \& Freedman, R. S. 2005, ApJ, 627, L69

Fortney, J. J., Saumon, D., Marley, M. S., Lodders, K., \& Freedman, R. S. 2006, ApJ, 642, 495

Gillon, M., et al. 2009a, A\&A, 506, 359

Gillon, M., et al. 2009b, A\&A, 496, 259

Gillon, M., et al. 2010, A\&A, 511, A3

Guillot, T., \& Havel, M. 2010, A\&A, in press (arXiv:1010.1078)

Harrington, J., Luszcz, S., Seager, S., Deming, D., \& Richardson, L. J. 2007, Nature, 447, 691

Hauschiltd, P. H., Allard, F., Ferguson, J., Baron, E., \& Alexander, D. R. 1999, ApJ, 525, 871

Hebb, L., et al. 2009, ApJ, 693, 1920

Hebb, L., et al. 2010, ApJ, 708, 224

Hebrard, G., et al. 2010, A\&A, 516, A95

Hubbard, W. B., Hattori, M. F., Burrows, A., Hubeny, I., \& Sudarsky, D. 2007, Icarus, 187,358

Husnoo, N., et al. 2010, submitted to MNRAS (arXiv:1004.1809)

Iro, N., \& Deming, D. 2010, ApJ, 712, 218

Knutson, H. A., Charbonneau, D., Allen, L. E., Burrows, A., \& Megeath, S. T. 2008, ApJ, 673, 526

Knutson, H. A., Charbonneau, D., Burrows, A., O'Donovan, F. T., \& Mandushev, G. 2009, ApJ, 691, 866

Knutson, H. A., et al. 2007, Nature, 447, 183

Knutson, H. A., et al. 2010, ApJ, 720, 1569

Lai, D., Helling, Ch., \& van den Heuvel, E. P. J. 2010, ApJ, 721, 923

Lanza, A. F., et al. 2009, A\&A, 493, 193

Li, S.-L., Miller, N., Lin, D. N. C., \& Fortney, J. J. 2010, Nature, 463, 1054

Machalek, P., McCullough, P. R., Burke, C. J., Valenti, J. A., Burrows, A., \& Hora, J. L. 2008, ApJ, 684, 1427

Morales-Calderon, M., et al. 2006, ApJ, 653, 1454

Okada, K., Aoyagi, M., \& Iwata, S. 2002, J. Quant. Spectrosc. Radiat. Transfer, 72,813

Pont, F., Knutson, H. A., Gilliland, R. L., Moutou, C., \& Charbonneau, D. 2008, MNRAS, 385, 109

Press, W. H., Teukolsky, S. A., Vetterling, W. T., \& Flannery, B. P. 1992, Numerical Recipes (Cambridge: Cambridge Univ. Press)

Rogers, J. C., Apai, D., Lopez-Morales, M., Sing, D. K., \& Burrows, A. 2009, ApJ, 707, 1707

Seager, S., Whitney, B. A., \& Sasselov, D. D. 2000, ApJ, 540, 504

Snellen, I. A. G., de Mooij, E. J. W., \& Albrecht, S. 2009, Nature, 459, 543

Snellen, I. A. G., de Mooij, E. J. W., \& Burrows, A. 2010, A\&A, 513, A76

Spiegel, D. S., Silverio, K., \& Burrows, A. 2009, ApJ, 699, 1487

Todorov, K., Deming, D., Harrington, J., Stevenson, K. B., Bowman, W. C., Nymeyer, S., Fortney, J. J., \& Bakos, G. A. 2010, ApJ, 708, 498 